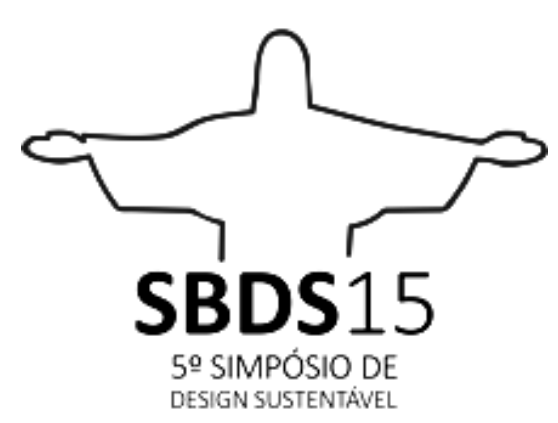

Rio de Janeiro, 11 de novembro a 13 de novembro de 2015

\title{
A INOVAÇÃO SOCIAL ORIENTADA PELO DESIGN: PERSPECTIVAS PARA CRIAÇÃO DE UMA PLATAFORMA HABILITANTE
}

\author{
Caio Marcelo Miolo de Oliveira \\ Universidade do Vale dos Sinos - UNISINOS \\ caiomarcelo.mo@gmail.com \\ Karine de Mello Freire \\ Universidade do Vale dos Sinos - UNISINOS \\ kmfreire@unisinos.br \\ Carlo Franzato \\ Universidade do Vale dos Sinos - UNISINOS \\ cfranzato@unisinos.br
}

\begin{abstract}
Resumo: O designer estratégico é identificado como potencial agente para catalisar inovações sociais. Através de suas competências de recombinar tecnologias, técnicas, disciplinas e propor diálogos entre os atores de uma determinada comunidade criativa/ organização colaborativa, pode empoderá-los a utilizar da criatividade que possuem para resolver os problemas existentes, ou gerar novas oportunidades que favoreçam a comunidade como um todo. Ao conceber instrumentos técnicos e organizacionais para tais fins, o designer estratégico favorece o desenvolvimento de plataformas habilitantes. Nesse contexto, o presente artigo trata sobre como o design estratégico pode desenvolver inovações sociais através da criação de plataformas habilitantes. Além de expandir o conhecimento sobre plataformas habilitantes, o principal objetivo deste trabalho é explorar como o design estratégico, apoiado na abordagem de Community Centered Design (CCD) e redes podem contribuir para o desenvolvimento de plataformas habilitantes. Como resultados, espera-se chegar a perspectivas preliminares de criação/ desenvolvimento de uma plataforma habilitante.
\end{abstract}

Palavras-chave: inovação social, plataformas habilitantes, design estratégico, Community Centered Design. 


\begin{abstract}
The strategic designer is identified as a potential agent to catalyze social innovations. Through their skills to recombine technologies, techniques, subjects and propose dialogues between the actors of a certain creative community / collaborative organization can empower them to use creativity having to solve problems or generate new opportunities that foster community as a whole. When designing technical and organizational instruments for such purposes, the strategic designer favors the development of enabling platforms. In this context, this article discusses how strategic design can develop social innovations by creating enabling platforms. In addition to expanding knowledge about qualifying platforms, the main objective of this study is to explore how the strategic design, based on the approach of Community Centered Design (CCD) and networks can contribute to the development of enabling platforms. As a result, it is expected to reach preliminary prospects for the creation / development of an enabling platform.
\end{abstract}

Keywords: social innovation, enabling platforms, strategic design, Community Centered Design.

\title{
1. INTRODUÇÃO
}

A atual era contemporânea caracteriza-se pela evolução constante dos meios de comunicação, de rapidez de inserções mercadológicas, de criação de novas tecnologias. Em meio a esta evolução, problemas sociais persistem e não conseguem ser devidamente solucionados (CASTELLS, 2010). Nesta linha, Touraine (2011), trata sobre a dificuldade de solucionar a crise atual existente, para atender simultaneamente os interesses econômicos e sociais de um país. Apesar de apontar a vigente incapacidade de resolver a crise social, Touraine (2011) traz resquícios de esperança, que podem partir de novos atores sociais: cidadãos, sujeitos portadores de direitos universais, com capacidades de se engajar nos conflitos, para reagir ao vazio social, perda de normas e aos conflitos sociais. Assim, é dado aos sujeitos sociais a responsabilidade de resistir e se opor às "tempestades deflagradas" que os ameaçam (TOURAINE, 2011, p.80).

O discurso de Touraine (2011) pede por uma ação social, que atue de forma sistêmica, envolvendo os próprios cidadãos a buscar formas de resolver ou melhorar a situação que não é local, ou tampouco simples. Neste âmbito, surgem novas formas de organização de atividades humanas, políticas, organizações não governamentais, a que Castells (2010) chama de sociedade em rede. As redes são impulsionadas pela forte evolução das tecnologias da informação, que "amplificam seu poder de forma infinita, à medida que os usuários apropriam-se dela e a redefinem" (CASTELLS, 2010, p.69). Tal poder das tecnologias da informação, de reconfiguração constante, é destacado como "aspecto decisivo em uma sociedade caracterizada por constante mudança e fluidez organizacional" (CASTELLS, 2010, p.109), que acabam por facilitar os processos de inovações decorridos em âmbitos organizacionais e sociais.

Para incentivar a união de competências, bem como explorar o poder que as redes possibilitam em âmbitos organizacionais, trazendo resultados para benefícios sociais, o designer estratégico é indicado como uma possível agente para promover um processo dialógico entre os vários atores envolvidos. A perspectiva do design estratégico, própria para ambientes organizacionais, sugere articulações entre atores, técnicas, tecnologias, conhecimentos e disciplinas para satisfazer diferentes necessidades e obter resultados de valor e com efeitos de sentido (ZURLO, 2010), agindo de forma sistêmica, considerando a comunicação das ações envolvidas (BENTZ, 2013). 
Devido às características do design estratégico em articulação com os vários atores, aproveitando das relações ativadas para desenvolver inovações que considerem os interesses, os valores coletivos, o impacto sociocultural resultante, Anna Meroni (2008a) afirma que o design estratégico está vinculado à inovação social. No âmbito da inovação social, o design utiliza suas sensibilidades, capacidades e habilidades para projetar estratégias que empoderem os membros de comunidades 1 a usar da criatividade que possuem para inovar em escala local, de forma que eles próprios solucionem os problemas sociais existentes ou gerem novas oportunidades (MANZINI, 2008a; MERONI, 2008a).

Como meios para facilitar o estabelecimento de conexões entre os membros da comunidade, bem como, diferentes atores e recursos que podem auxiliar no desenvolvimento de inovações sociais, estão as plataformas habilitantes2: "um número de iniciativas habilitantes capazes de suportar uma variedade de organizações colaborativas3" (MANZINI, 2008a, p.86). Corroborando, Murray et al. (2010, p.138) identificam como intermediações para "dar às pessoas as ferramentas e recursos de que precisam para se organizarem". Segundo Manzini (2008a), as iniciativas que compõem as plataformas habilitantes compreendem intervenções estruturadas formadas por produtos/serviços que possam ser aplicados em diferentes comunidades criativas/organizações colaborativas, com finalidades, como: facilitar a comunicação entre os seus membros, o acesso de pessoas a elas, tornar mais fluído e fácil o desenvolvimento dos processos de inovações social decorrentes.

Neste âmbito explicitado, ao atuar junto às comunidades/organizações de modo a capacitá-las a trabalhar colaborativamente para solucionar problemas, ou criar novas oportunidades para benefício comum (MERONI, 2008a), apóia-se na abordagem de Community Centered Design (CCD), termo cunhado por Meroni (2008a), onde o objetivo principal do design está em conceber soluções projetuais junto aos membros das comunidades. Segundo Cantú el al. (2012) para trabalhar com CCD é necessário ao designer obter duas competências principais: desenvolver relações de empatia com os membros da comunidade e com o contexto, de modo que facilite a interação e desenvolvimento das soluções projetuais; e a segunda, elaborar ferramentas para projetar com e para esta comunidade, habilitando o co-design, permitindo que os membros da comunidade apliquem seus conhecimentos e habilidades para contribuir com os projetos em questão.

Por conseguinte, devido as qualidades do design estratégico (apoiado na abordagem de CCD) de trabalhar em redes, inter-relacionando diferentes atores, técnicas, tecnologias, conhecimentos e disciplinas para desenvolver inovações sociais, o presente artigo tem como objetivos apresentar o conceito de plataformas habilitantes e explorar as abordagens de design estratégico, CCD e redes para identificar possíveis contribuições ao desenvolvimento dessas plataformas.

\section{REDES}

O atual período em que vivemos é marcado pela revolução tecnológica, que ocorre com tamanha progressão e poder de dissipação que acaba por remodelar as estruturas sociais, formando novas formas de relações entre a economia, o Estado e a sociedade. Neste período de revolução tecnológica o conhecimento e a informação não são mais centralizados em alguma determinada área dessa tríade, mas disseminados em rede, processados e comunicados,

\footnotetext{
${ }^{1}$ Conforme Manzini (2008a, p.61) as comunidades criativas "são indivíduos e comunidades que agem para resolver seus problemas ou criar novas oportunidades".

${ }^{2}$ Murray et al (2010) chamam de plataformas de inovação, porém com o mesmo sentido tratado por Manzini (2008a).

${ }^{3}$ Os casos promissores de comunidades criativas tornam-se organizações colaborativas (MANZINI, 2008a, p.70).
} 
possibilitando a geração de novos conhecimentos, "em um ciclo de realimentação cumulativo entre a inovação e o uso" (CASTELLS, 2010, p.69). E devido ao potencial de reconfiguração das tecnologias de informação, as redes tornam-se determinantes para a sociedade, que é marcada por constantes mudanças e fluidez organizacional (CASTELLS, 2010). A partir da perspectiva de Castells (2010), percebe-se que as redes, disseminadas pelas tecnologias da informação, não são independentes e autosuficientes por si só, mas o que determina seu valor são as formas de uso, de transformações que elas possibilitam

Ao tratar sobre redes, é importante destacar que elas não englobam apenas interações por mediação tecnológica (por computadores, celulares, internet, etc) mas envolve também relações diretas entre as pessoas, entre organizações; não havendo portando distinções entre coisas e pessoas nas redes, pois tudo que contribui para seu desenvolvimento é considerado um ator (LÉVY, 2011). E nesse âmbito, estão os sistemas distribuídos, tratados por Manzini (2008a): "uma teia de elementos interconectados" que são capazes de funcionar simultaneamente e autonomamente, trabalhando para alguma finalidade comum (MANZINI, 2008a, p.94). Segundo o autor, as relações simultâneas dos sistemas distribuídos rompe com a estrutura formal de sistemas tradicionais (hierárquicos ou centralizadores), tornando-se sistemas horizontais, que possibilitam uma relação entre comunidades e seus recursos tecnológicos de modo mais acessível e assim, mais democrático de gerenciá-los.

Outro valor dos sistemas distribuídos abordados por Manzini (2008a) é o desenvolvimento de uma inteligência distribuída4, ou seja, o conhecimento pode ser melhor difundido entre os componentes das redes, possibilitando maiores chances de produzir inovações. Conforme Lévy (2011) a lógica desta abordagem consiste no compartilhamento de saberes, obtida a partir da valorização dos saber individuais. Com determinados dados fornecidos por outros atores da rede, pode-se interpretá-los, unir com seus próprios conhecimentos e transformar em outros conhecimentos, podendo resultar em inovações. Toda concepção e inovação corresponde em utilizar de maneira original elementos que já existem (LÉVY, 2011; CASTELLS, 2010).

Nesse contexto explicitado, para incentivar a união de competências, explorar o potencial das redes, catalisando um grande número de pessoas a promoverem inovações no âmbito social, o designer estratégico é mostrado como um possível agente para tal processo e intermediação. No subtítulo a seguir é apresentado o potencial do design estratégico e sua articulação no âmbito de inovações sociais.

\section{DESIGN ESTRATÉGICO E INOVAÇÃO SOCIAL}

O design estratégico é definido como um processo projetual ou fenômeno próprio para atuar em ambientes coletivos na busca por resolver problemas complexos, melhorar situações ou gerar soluções inovadoras e efeitos de sentido (dimensão de valor para alguém), concretizando o resultado em um produto-serviço, uma representação visível da estratégia (ZURLO, 2010). O design estratégico caracteriza-se por ser um processo que permite e propõe diversos pontos de vista, modelos interpretativos articulados e variadas disciplinas, o que o coloca como uma abordagem própria para a complexidade da sociedade, sejam elas de ordem técnica, processual ou organizacional (ZURLO, 2010).

O designer estratégico analisa os problemas a partir do "pensamento complexo" (ZURLO, 2010), ou seja, analisa os vários fatores relacionados, para posteriormente hierarquizar o que é importante (MORIN, 2006). Nesse sentido, o designer consegue interpretar a complexidade, capturando dela, estruturas de sentido, a fim de encontrar caminhos resolutivos (ZURLO, 2010).

\footnotetext{
${ }^{4}$ Tratada como "inteligência coletiva” por Lévy, (2011).
} 
Outra característica processual do design estratégico, é que ao considerar a complexidade e articular com diferentes disciplinas, ele também promove ações dialógicas entre vários atores, sendo um facilitador do processo projetual e agente de modificação das realidades existentes (ZURLO, 2010). Nesse sentido, através de articulação com os vários atores, aproveitando das relações ativadas para canalizá-las à conpecção de inovações para o bem comum de determinada comunidade/organização, considerando os interesses coletivos, o impacto sociocultural resultantes, Meroni (2008a) destaca que o design estratégico está diretamente relacionado à inovação social.

Antes de explicitar como o design estratégico atua para propiciar desenvolvimento de inovações sociais, é necessário definir o que é inovação social, sob a perspectiva dos autores alinhados a essa matriz teórica. Inovação social é caracterizada por ser "uma mudança no modo como indivíduos ou comunidades agem para resolver seus problemas ou criar novas oportunidades [...] geralmente emergindo através de processos organizacionais [...]" (MANZINI, 2008a, p.62). Nesse sentido, o designer é indicado potencial agente de inovação. $O$ designer cada vez mais se distancia da atividade tradicional de design, vinculada à criação de produtos, identidades visuais, para tornar-se "designer social", um colaborador que ajude profissionalmente esta comunidade na tomada de decisões estratégicas e projeção de seu futuro (MERONI, 2008b, p.7).

No entanto, mais que facilitar o processo de capacitação e mudança de comportamentos, o designer adquire a função de catalisar5 o percurso projetual, pois possui capacidades para imaginar e influenciar comportamentos, trazendo seu ponto de vista profissional, sua experiência, "levando e elaborando o melhor do presente e transformando-o em uma mudança de paradigma para o futuro" (MERONI, 2008b, p.30). Para tanto, o designer articula entre diferentes disciplinas, técnicas, estratégias para desenvolver artefatos que facilitem esta mediação (MERONI, 2008b).

Nesse sentido, Manzini (2008a) trata sobre o modelo de inovação peer-to-peer, que estabelece uma relação de modo paritário horizontal com os usuários envolvidos, para construção de organizações colaborativas. Esta perspectiva demanda do designer promover colaboração de forma co-criativa, participação na construção de cenários compartilhados; e combinar serviços e produtos já existentes para suportar a específica comunidade criativa (MANZINI, 2008a).

Por conseguinte, neste âmbito de introdução de agentes de design para auxiliar as organizações colaborativas/ comunidades criativas, Manzini (2008a) destaca a importância de aumentar a acessibilidade, a eficácia das ações criativas e torná-las capazes de serem difundidas, e replicadas. Para isto, o autor afirma que mesmo não sendo possível planejar completamente as inovações sociais nestas organizações e comunidades é possível facilitar sua criação ou desenvolvimento através de intervenções de suporte, as soluções habilitantes e plataformas habilitantes, explicadas a seguir.

\subsection{Soluções e plataformas habilitantes}

Para esclarecer o que são plataformas habilitantes é necessário inicialmente explorar o conceito de soluções habilitantes, visto que o conjunto destas compõem as plataformas habilitantes (MANZINI, 2008a; FRANQUEIRA, 2010). As soluções habilitantes são identificadas por Meroni e Sangiorgi (2011, p.148) como "soluções técnicas e artefatos para habilitar as pessoas a cooperarem para alcançar um determinado resultado num dado contexto". Estas soluções são reconhecidas por Manzini (2008a) por serem próprias para o âmbito organizacional, compreendendo assim produtos, serviços, formas de comunicação, entre outras ações, que auxiliem as comunidades criativas e organizações colaborativas a serem acessíveis, eficazes, e por

\footnotetext{
${ }^{5}$ Compreende-se que "catalisar" refere-se a acelerar as ações de um processo, habilitando as relações.
} 
fim, replicáveis. Acessíveis, de modo que os membros da comunidade tenham ferramentas, infraestrutura e estratégias para compensar a demanda de tempo e dedicação necessários para atuar nestas comunidades ou organizações; eficazes de modo a compensar os esforços em resultados positivos; e a replicáveis, que possa ser aplicada em outras comunidades e organizações (MANZINI, 2008a).

As soluções habilitantes são mencionadas ainda como atividades para encorajar a participação de membros de comunidades e organizações de modo colaborativo (MANZINI, 2008b). Nesse sentido, o autor destaca a importância de estabelecer relações para aprendizado social, que envolve acabar com soluções desabilitantes, ou seja, incentivar "a lógica indutiva individual e criativa social" (MANZINI, 2008b, p.17), de maneira que se desabilite costumes anteriores de comodismo e passividade dos membros dessas comunidades. Portanto, para projetar soluções habilitantes, mostra-se necessário agir inicialmente na mudança de comportamento das pessoas.

Dentre as questões que precisam ser resolvidas ou melhoradas por intermédio de soluções habilitantes estão (MANZINI, 2008a): promoção de estratégias de comunicação motivadoras e capazes de fornecer os conhecimentos necessários aos membros da comunidade; tentativa de redução do total de tempo e espaço requeridos; facilitação do processo de constituição de comunidades e de inclusão de agentes que desejam ajudá-las; ações para estimular e desenvolver habilidades dos membros.

Apesar das soluções habilitantes requererem também tecnologias comunicacionais e mesmo personalizadas, Manzini (2008a) destaca que para atuar de forma colaborativa, as comunidades e organizações utilizam e necessitam de tecnologias basicamente comuns, a partir de produtos e serviços disponíveis no mercado, como telefones, computadores e internet. A importância para ele está na maneira como as tecnologias são exploradas.

Depois de explicitado o que são soluções habilitantes, pode-se caracterizar as plataformas habilitantes: um conjunto de soluções habilitantes (FRANQUEIRA, 2010), que segundo Manzini e Jégou (2003, p.111) trata-se de "um sistema que fornece instrumentos cognitivos, técnicos e organizacionais para habilitar usuários a alcançar resultados, usando suas competências e habilidades para o melhor proveito". Corroborando, Warnke e Cagnin (2006, p.173) identificam as plataformas habilitantes como "um sistema de elementos materiais e imateriais (tais tecnologias, infra-estruturas, padrões jurídicos e governativos e elaboração de políticas) concebidas para gerar um contexto favorável para as comunidades criativas e casos promissores".

Manzini e Jégou (2003, p.111) identificam que o conceito de uma plataforma habilitante compreende "a possibilidade de ver a tecnologia como um sistema que aumenta e fortalece oportunidades individuais e coletivas" (p.112). Estas ferramentas e tecnologias exploradas nas plataformas habilitantes devem ser utilizadas pelos usuários de forma sustentável, ou seja, de modo que consigam por conta própria utilizá-las (MANZINI e JÉGOU, 2003). Isto quer dizer que o designer, nesses contextos deve projetar para o que Cantú (2012) chama de o pós-design. 0 designer, ao estruturar e construir projetos junto às comunidades - sejam produtos, serviços ou sistemas - precisa projetá-los de modo que sejam auto-sustentáveis, funcionando sem a presença do designer após a projetação; isto significa projetar o pós-design. Percebe-se nesse ponto uma convergência com a projetação de plataformas habilitantes, que de certo modo, é um pós-design, que precisa ser planejado.

Assim preparar para o pós-design significa que o trabalho dos designers é envolver nãodesigners a conceber ideias futuras, construindo sobre as capacidades dos membros das comunidade, fornecendo o apoio adicional para cobrir todas as áreas de fraqueza, integrando, conhecimentos e habilidades anteriormente ausentes (CANTÚ, 2012). Para prever as possíveis 
dificuldades, o design pode possivelmente trabalhar com a construção de cenários. A construção de cenários cria um contexto no qual se imagina usuários lidando com produtos e serviços potenciais e experimentando-os para fazer emergir novas ideias (ZURLO, 2010). Os cenários criados devem ser pertinentes, baseados na realidade sistêmica, visando contribuir socialmente e considerar os agentes envolvidos, o fluxo de eventos e as consequências de cada cenário criado (MORELLI, 2002).

Considerando a necessidade de envolver os membros da comunidade no projeto estratégico de uma plataforma habilitante e por conseguinte, planejar o pós-design, mostra-se pertinente explorar a abordagem de Community Centered Design (CCD).

\subsection{Community Centered Design}

O Community Centered Design (CCD) é uma abordagem projetual proposta por Meroni (2008a), na qual se tem como objetivo atender necessidades de maneira coletiva ou qualificar o bem-estar de comunidades, através da compreensão sobre comportamentos e necessidades destas. As soluções originadas são criadas em colaboração com os membros da comunidade, que são os especialistas do seu contexto cotidiano e possuem habilidades que podem ser usadas em benefício da comunidade, (MERONI, 2008a; CANTÚ, 2012), deste sistema distribuído, detentor de uma inteligência distribuída, conforme já abordado neste artigo no subtítulo de redes.

A partir da inserção da comunidade no processo projetual, definem-se plataformas e ferramentas para incentivar a participação criativa destas pessoas (COTTAM e LEADBEATER, 2004) a "desenvolver novas habilidades e competências para que não-designers participem da definição, implementação dos projetos, e criação de soluções adequadas para os específicos usuários e contextos locais" (CANTÚ, 2012, p.173). Seriam estas portanto as soluções habilitantes, que em conjunto formam uma plataforma habilitante.

Porém, para se definir estratégias e ferramentas para incentivar participação dos membros da comunidade, facilitar as interações e desenvolvimento projetual, Cantú et al. (2012) indicam que é necessário ao designer trabalhar com duas competências principais: desenvolver relações de empatia com os membros da comunidade e com o contexto, de modo à facilitar a interação e desenvolvimento das soluções projetuais (design empático); e a segunda, elaborar ferramentas para projetar com e para esta comunidade, de forma que se habilite o co-design, permitindo que os membros da comunidade apliquem seus conhecimentos e habilidades para contribuir com o projeto em questão.

Design empático é uma área de conhecimento já difundida, que propõe o desenvolvimento de conceitos e produtos a partir da perspectiva do usuário. Para obter estas informações sobre os usuários, mostra-se necessário "usar os sapatos dos outros", ou seja, colocar-se no lugar do usuário, a fim de perceber o que os motiva, como é o contexto em que ele vive, quais seus hábitos, suas experiências cotidianas (OUDEN, 2012). No CCD, empatia envolve estas mesmas questões, compreender o que sentem e vivem as pessoas desta comunidade, aprender a partir da observação e reflexão, alcançando conhecimentos ocultos sobre suas necessidades e desejos, que possibilitarão insights projetuais (CANTÚ, 2012). Como estratégias para estabelecer empatia, está inicialmente o diálogo (CANTÚ, 2012) ou conversas empáticas, como é tratado por Meroni e Sangiorgi (2011). Segundo as autoras, o ponto inicial das conversas empáticas começa com a identificação do que precisa ser compreendido sobre os relacionamento atuais e passados das partes interessadas (pessoas da comunidade). Para isso, é necessário planejar previamente de que forma ocorrerão as conversas empáticas, podendo iniciar por perguntas, ou através de artefatos que facilitem a comunicação. Como estratégias de artifícios artísticos para incutir uma maior empatia, como, visualização fotografias dos usuários, desenhos e escritas feitos por eles, que 
podem ser até mais eficazes do que diálogos diretos com estas pessoas (MCGINLEY e DONG, 2011).

Além dos diálogos (conversas empáticas), as atividades de co-design também são pertinentes para geração de empatia (CANTÚ, 2012). O co-design, refere-se ao processo de design, em que designers trabalham junto com pessoas não designers, orientando-as e treinado-as para desenvolver algum determinado projeto em conjunto (SANDERS e STAPPERS, 2008, p.6). Para atuar em comunidades e utilizar atividade de co-design, Cantú (2012, p.173) identifica alguns exemplos de ferramentas e capacidades que o designer pode desenvolver, como: desenvolvimento de protótipos simples para tornar visíveis as ideias e facilitar a interação; habilidade de contar histórias (storytelling), que podem ser realizada com mediação dos protótipos rápidos já citados.

O desenvolvimento das atividades de co-design e design empático visam fornecer conhecimentos tácitos e explícitos sobre os membros da comunidade e o contexto existente. Ambos os tipos de conhecimentos estão interligados, estando inevitavelmente em cada ação realizada (CANTÚ, 2012). Os conhecimentos explícitos citados são aqueles percebidos com base nas respostas às perguntas realizadas, onde são nítidas as informações. Quanto a isto, a autora indica deixar perguntas "em aberto", ou seja, respostas diferentes do "sim" e "não" apenas, tentando estender as conversas, tornando-as mais informais, poderá possibilitar respostas mais sinceras e espontâneas. Já os conhecimentos tácitos são aqueles percebidos nos comportamentos, humores e sentimentos que não são expressos "verbalmente" nas atividades de co-design ou entrevistas realizados, por exemplo, mas são percebidos através de observações.

Por conseguinte, devido à abordagem inclusiva e constituinte de ferramentas para efetivar relações de empatia e co-design, a abordagem de CCD, acredita-se que seja pertinente uma convergência disciplinar para contribuir para o desenvolvimento de plataformas habilitantes.

\section{PERSPECTIVAS PROJETUAIS PARA CRIAÇÃO DE UMA PLATAFORMA HABILITANTE}

Inicialmente é válido ressaltar que uma plataforma habilitante, ao ser compreendida por um sistema que fornece instrumentos cognitivos, técnicos e organizacionais para habilitar seus membros (MANZINI e JÉGOU, 2003), está impreterivelmente em rede, pois todos os componentes humanos e não humanos como técnicas, tecnologias, também fazem parte da rede, associada às tecnologias da informação.

Neste âmbito, o designer estratégico através de suas competências de recombinar tecnologias, técnicas, disciplinas e propor diálogos entre os atores da rede pode estimular a inteligência distribuída, e portanto, empoderar os membros desta comunidade criativa / organização colaborativa a utilizar de sua criatividade para resolver os problemas existentes ou gerar novas oportunidades que favoreçam a comunidade como um todo. Ao favorecer a comunidade com um todo, o designer estratégico mostra-se como um catalisador e articulador da inovação social.

Para propor diálogos com as comunidades, incentivar o potencial de seus membros (habilitá-los), o designer estratégico pode se valer da abordagem do CCD. O CCD propõe o desenvolvimento de duas competências possivelmente primordiais para facilitar a interação e desenvolvimento das soluções projetuais: as relações de empatia e co-design, que visam fornecer conhecimentos tácitos e explícitos sobre os membros da comunidade, bem como do contexto existente, e fornecer ferramentas para projetar com e para esta comunidade, permitindo que os membros da comunidade apliquem seus conhecimentos e habilidades para contribuir com o projeto em questão (CANTÚ, 2012). 
Por conseguinte, o designer estratégico, com suas capacidades interdisciplinares, de combinação de técnicas, tecnologias, de proposição de diálogos, de tornar tangíveis estratégias criadas, de exploração do saber de todos da rede, mostra-se necessário e imprescindível o desenvolvimento de relações de empatia e co-design. Através destas ações, mais que um facilitador de inovações sociais, ele torna-se um agente catalisador, que desencadeia e acelera o princípio e desenvolvimento destes processos. E assim, ao realizar estas articulações, o designer acaba por favorecer o desenvolvimento de uma plataforma habilitante, um sistema que fornece instrumentos cognitivos, técnicos e organizacionais para habilitar usuários a alcançar resultados, que de certo modo, projetados de modo que sejam auto-sustentáveis, funcionando sem a presença do designer após a projetação.

Como resultado desta plataforma habilitante está o desenvolvimento de uma comunidade autosuficiente, acessível, eficaz e de modo que as estratégias desenvolvidas possam ser replicáveis em outras comunidades criativas e organizações colaborativas.

\section{CONCLUSÃO}

Os objetivos propostos neste artigo foram de apresentar o conceito de plataformas habilitantes e identificar possíveis contribuições teóricas em redes, design estratégico e da abordagem de CCD. Pôde-se compreender através do presente estudo as seguinte questões:

1. As plataformas habilitantes mostrarem-se como estratégia pertinente para inovação social. Uma estratégia que visa fornecer ferramentas, técnicas e tecnologias de aprendizado social, para aumentar e fortalecer habilidades de comunidades e organizações, de modo que seus membros consigam por conta própria utilizá-las e a partir disto, conseguir resolver seus problemas ou criar novas oportunidades para a comunidade/organização.

2. Ao articular com os vários atores, empoderando os membros das comunidades e organizações a utilizar suas habilidades e conhecimentos através de estratégias codesign, o designer estratégico estimula o desenvolvimento de uma inteligência distribuída. Através do incentivo ao compartilhamento de saberes em rede, o designer favorece que os membros da comunidade/organização, assimilem conhecimentos de outros atores e recombinem essas informações, transformandoas em novos conhecimentos, podendo resultar em inovações. Relacionando às plataformas habilitantes, a inteligência distribuída, mostra-se como atributo imprescindível e inseparável a elas, visto que o conhecimento precisa ser disseminado dentro desta rede para que os membros consigam fortalecer suas habilidades e agir por conta a favor da comunidade ou organização.

3. Através da abordagem inclusiva e constituinte de ferramentas para efetivar relações de empatia e co-design, habilitando não-designers a participarem de definições e implementações projetuais, o Community Centered Design foi percebido como abordagem relevante para projetar com a comunidade e facilitar o processo de desenvolvimento de plataformas habilitantes.

4. Assim como tratado na abordagem de Community Centered Design, o desenvolvimento de plataformas habilitantes também envolve projetar o pósdesign. Ao conceber ferramentas, técnicas e tecnologias para estruturar e construir projetos junto às comunidade e organizações, o designer precisa projetá-las de modo que sejam auto-sustentáveis, funcionando sem a presença do designer após a projetação. E nesse sentido, as abordagens de design empático e co-design trazidas no Community Centered Design mostram-se relevantes por fornecer 
conhecimentos tácitos e explícitos sobre a comunidade, possibilitar a construção sobre suas capacidades, visando fornecer apoio adicional, conhecimentos e habilidade para cobrir áreas de fraqueza que possuem ou podem existir em determinada comunidade ou organização.

Por fim, com base nas perspectivas projetuais explicitadas neste trabalho, pôde-se chegar a algumas contribuições teóricas, que precisam ser melhor aprofundadas e verificadas em termos práticos.

\section{REFERÊNCIAS}

BENTZ, Ione. Processos de significação para projetos de design. In: PARODE, Fábio; BENTZ, lone (Org.). Cadernos de Cultura e Estética Contemporânea. Porto Alegre: Entremeios, 2013.

CANTÚ, Daria. Ideas Sharing Lab. Community Centred Design for Multifuncional and Collaborative Food Services. Doctoral Thesis, Politécnico Di Milano, FEB. 2012.

CANTÚ, Daria; CORUBOLO, Marta; SIMEONE, Giulia. A Community Centered Design approach to developing service prototypes. 2012. Disponível em: < http://www.servdes.org/ >. Acesso em: 20 jan. 2015.

CASTELLS, M. A sociedade em rede. São Paulo: Paz e Terra, 2009.

COTTAM, H.; LEADBEATER C. Health: Co-creating Services. London, Design Council, 2004.

FRANQUEIRA, Teresa. Creative Places for Collaborative Cities. Universidade de Aveiro. Milan, Italy, 2010. Disponível em: < http://aveiro.academia.edu/teresafranqueira > . Acesso em: 30 jul. 2015.

LÉVY, P. As tecnologias da inteligência: O futuro do pensamento na era da informática. Lisboa: Instituto Piaget, 1992.

LÉVY, P. A inteligência coletiva: para uma antropologia do ciberespaço. São Paulo: Edições Loyola, 2011.

MANZINI, E; JÉGOU, F. Sustainably everyday: scenarios of urban life. Milano: Edizioni Ambiente, 2003.

MANZINI, E. Design para inovação social e sustentabilidade: comunidades criativas, organizações colaborativas e novas redes projetuais. Rio de Janeiro: E-Papers, 2008a.

MANZINI, E. Design context: enabling solutions for sustainable urban everyday life. In: MANZINI, Ezio; WALKER, Stuart ; WYLANT, Barry. Enabling Solutions for Sustainable Living: A Workshop. Calgary: University of Calgary Press, 2008b.

MCGINLEY, Chris; DONG, Hua. Designing with Information and Empathy: Delivering Human Information to Designers. In: The Design Journal VOLUME 14, ISSUE 2 PP 147-150, 2011.

MERONI, Anna. Strategic design: where are we now? Reflection around the foundations of a recent discipline. Strategic Design Research Journal, v.1, n.1, Dec 1, p.31-38, 2008a.

MERONI, Anna. Strategic Design to take care of the territory. Networking Creative Communities to link people and places in a scenario of sustainable development. In: P\&D Design 2008, 80 Congresso Brasileiro de Pesquisa e Desenvolvimento em Design. São Paulo, 2008b.

MERONI, Anna; SANGIORGI, Daniela. Design for services. Gower Publishing, 2011. 
MORELLI, Nicola. Designing Product/Service Systems: A Methodological Exploration. In: Design Issues, v.18, n.3, p.3-17, 2002. Disponível em: <http://www.jstor.org/stable/1512062>. Acesso em 11 out. 2014.

MORIN, Edgar. Introdução ao pensamento complexo. Porto Alegre: Sulina, 2006.

MURRAY, R; CAULIER-GRICE, J; MULGAN, G. The open book of social innovation. London: The Young Foundation \& NESTA, 2010.

OUDEN, Elke den. Innovation Design: Creiating value for people, organizations and society. 2012.

SANDERS, Elizabeth B-N; STAPPERS, Pieter Jan. Co-creation and the new landscapes of design. CoDesign, v.4, n.1, p-5-18, mar. 2008. Disponível em: <http://www.tandfonline.com/doi/abs/10.1080/15710880701875068>. Acesso em 10 jun. 2015.

TOURAINE, A. Após a crise. Rio de Janeiro: Vozes, 2011.

WARNKE, Philine; CAGNIN, Cristiano. Articulation with the macro-context. From the micro to the macro level: Methodological considerations. In: JÉGOU, F; MANZINI, E. Collaborative services: social innovation and design for sustainability. Milano: Edizioni Poli.design. 2006.

ZURLO, Francesco. Design Strategico. In: XXI Secolo, vol. IV, Gli spazi e le arti. Roma: Enciclopedia Treccani, 2010. 